\title{
Functionals of Type 3 as Realisers of Classical Theorems in Analysis
}

\author{
Dag Normann \\ Department of Mathematics, The University of Oslo, P.O. Box 1053, Blindern N-0316 \\ Oslo, Norway, dnormann@math.uio.no
}

\begin{abstract}
We investigate the relative computational strength of combinations of four higher order functionals, the jump and hyperjump seen as functionals of type 2 and realisers for the compactness of Cantor space and the Lindelöf property of Baire space seen as functionals of type 3 . We compare them with the closure operator for non-monotone inductive definitions of sets of integers, also seen as a functional of type 3 .
\end{abstract}

\section{Background}

The current note is a spin-off from a joint project with Sam Sanders, based on his original initiative. In [15], Sanders used the Kohlenbach-inspired axiomatisation of nonstandard analysis from [1] to obtain results in computability theory from nonstandard analysis. His proof-theoretical analysis of the nonstandard proofs of Weak König's Lemma and the less known Weak Weak König's Lemma, combined with methods of term extraction, led to the discovery of two classes of type three functionals, the $\Theta$-functionals (that will be discussed further in this note), and the $\Lambda$-functionals. For the latter, see also [11-13].

Sanders got the author of this note interested in the computational strength of these objects, and a fruitful cooperation started. As a preliminary result, it was established that these objects are genuinely of type 3 , no $\Theta$ - or $\Lambda$-functional is computable, in the sense of Kleene, in any object of type 2. Moreover, it became clear that there will be $\Theta$ - and $\Lambda$-functionals that are countably based. A functional $\Psi$ of type 3 is countably based if the value of $\Psi(F)$ will be determined from the restriction of $F$ to a countable set. The initial investigations of countably based functionals were described by John P. Hartley [6-8]. At the time, there were few natural examples beyond the Superjump. The fan functional and the Gandy-Hyland functional are important objects of type three, but they are nowadays mainly considered as functionals that are restricted to the class of continuous objects of type 2. Our project now has shown that many natural functionals of type 3 belong to the class of countably based functionals.

Next, it became clear that there are $\Theta$ - and $\Lambda$-functionals that are sequential in the sense that the value can be "computed" via a transfinite recursive procedure. It also became clear that these functionals are intimately related to some classical theorems in mathematics, such as the Heine-Borel theorem and the Vitali covering theorem. In particular, we observed that the sequential transfinite 
procedure used to construct one particular $\Theta$-functional turned out to be an almost line-to-line transition of a construction due to Borel, [2]. These connections are to some extent explored in [14], are brought further in [16] and will also be investigated in the continuation of our project.

We now see $\Theta$ as a realiser for the compactness of the Cantor space, and we have introduced another class of functionals, the $\Xi$-functionals, that can be seen as realisers of the Lindelöf property of the Baire space $\mathbb{N}^{\mathbb{N}}$. The technical aim of this note is to show how the computational strength of these objects are related, and also to link this to the complexity of (non-monotone) inductive definitions of subsets of $\mathbb{N}$. As a part of this, we will use the Borel-method to produce a realiser for the Lindelöf property, and indirectly, give a kind of Reverse Mathematics characterisation of the strength of the Borel method from 1895.

\section{The Five Functionals}

\subsection{Two Functionals of Type 2}

We will consider the functional $\mu$, known as Feferman's $\mu$, and the Suslin functional $\mathrm{S}$ as follows:

$$
\begin{aligned}
& \mu(f):=\left\{\begin{array}{l}
0 \text { if } f \text { is constant zero } \\
\text { the least } a \text { such that } f(a)>0 \text { otherwise. }
\end{array}\right. \\
& S(f):=\left\{\begin{array}{l}
0 \text { if } \forall g \exists n(f(\bar{g}(n))=0) \\
1 \text { if } \exists g \forall n(f(\bar{g}(n))>0)
\end{array}\right.
\end{aligned}
$$

where $f$ and $g$ are functions on $\mathbb{N}$, and $\bar{g}(n)$ is the number $\langle g(0), \ldots, g(n-1)\rangle$, which we identify with the corresponding sequence.

We use $\mu$ instead of $\exists^{2}$, also known as ${ }^{2} E$ : modulo full Kleene computability, they are equivalent, while in fragments like e.g. Gödel's T, $\mu$ is more expressive that $\exists^{2}$. Arithmetical definitions can be transformed to terms using $\mu$, and our use of $\mathrm{S}$ will mainly be to isolate the well-founded segment of a relation.

\subsection{Specifications for Realisers of Type 3}

Without digging too deep into the theory of realisers, we can say that a realiser of a theorem of the form $\forall x \exists y(A(x) \rightarrow B(x, y))$ is a function $\phi$ that maps (a realiser for) an $x$ satisfying $A$ to some $y$ such that $B(x, y)$ (and a realiser for this). We analyse the complexity of realisers of this kind for two classical theorems:

1. Compactness of the Cantor space

For each $x \in C$, let $O_{x} \subseteq C$ be an open set containing $x$. Then there is a finite set $\left\{x_{1}, \ldots, x_{n}\right\} \subseteq C$ such that $C \subseteq O_{x_{1}} \cup \cdots \cup O_{x_{n}}$.

2. The Lindelöf Property of the Baire space

For each $x \in \mathbb{N}^{\mathbb{N}}$, let $O_{x} \subseteq \mathbb{N}^{\mathbb{N}}$ be an open set containing $x$. Then there is a sequence $\left\{x_{i}\right\}_{i \in \mathbb{N}}$ in $\mathbb{N}^{\mathbb{N}}$ such that $\mathbb{N}^{\mathbb{N}} \subseteq \bigcup_{i \in \mathbb{N}} O_{x_{i}}$. 
In [14] we discuss how these statements relate to the classical theorems known as the Heine-Borel theorem and the Lindelof property for subsets of $\mathbb{R}$. Here we will consider equivalent, styled versions suitable for interpretations over the standard typed structure of functionals with base type $\mathbb{N}$.

For the sake of simplicity, we will identify a finite sequence $s$ of non-negative integers with its sequence number $\langle s\rangle$. If $s$ is a finite binary sequence of length $n$, we let $C_{s}$ be the set of binary functions $f$ with $s=\bar{f}(n)$, while if $s$ is a finite sequence of non-negative numbers, still of length $n$, we let $B_{s}$ be the set of $f: \mathbb{N} \rightarrow \mathbb{N}$ such that $\bar{f}(n)=s$. Any $F: \mathbb{N}^{\mathbb{N}} \rightarrow \mathbb{N}$ will define open coverings of $C$ resp. $\mathbb{N}^{\mathbb{N}}$. They are $\left\{C_{\bar{f}(F(f))} \mid f \in C\right\}$ resp. $\left\{B_{\bar{f}(F(f))} \mid f \in \mathbb{N}^{\mathbb{N}}\right\}$. Moreover, any general cover allocating an open set $O_{x}$ containing $x$ to each $x$ in the spaces $C$ or $\mathbb{N}^{N}$ can, with an arithmetical construction, be replaced by one obtained from a functional as above, without simplifying the task of verifying compactness or the Lindelöf property.

We can then define what we mean with a realiser $\Theta$ for the compactness of $C$ or a realiser $\Xi$ for the Lindelöf property of $\mathbb{N}^{\mathbb{N}}$ by

\section{Definition 1.}

$\Theta$ An instance of $\Theta$ will be a functional, that we also denote $\Theta$, of type 3 such that if $F: C \rightarrow \mathbb{N}$, then $\Theta(F)$ is a finite sequence $f_{1}, \ldots, f_{n}$ in $C$ such that the $C_{\bar{f}_{i}\left(F\left(f_{i}\right)\right)}$ 's cover $C$.

$\Xi$ An instance of $\Xi$ will be a functional, that we also denote $\Xi$, of type 3 such that if $F: \mathbb{N}^{\mathbb{N}} \rightarrow \mathbb{N}$, then $\Xi(F)$ is an infinite sequence $\left\{f_{i}\right\}_{i \in \mathbb{N}}$ from $\mathbb{N}^{\mathbb{N}}$ such that $\left\{B_{\bar{f}_{i}\left(F\left(f_{i}\right)\right)}\right\}_{i \in \mathbb{N}}$ covers $\mathbb{N}^{\mathbb{N}}$.

It is routine to rewrite these definitions to fit pure type 3 , but for the sake of readability, we prefer not to do this routine.

The definitions of $\Theta$ and $\Xi$ may look similar, but there is an important difference: while checking if a finite set $s_{1}, \ldots, s_{n}$ of binary sequences induce a finite covering of $C$ is computable at a low level, the set of countable sequences $\left\{s_{i}\right\}_{i \in \mathbb{N}}$ inducing a countable covering of $\mathbb{N}^{\mathbb{N}}$ will be complete $\Pi_{1}^{1}$. This is reflected in Thm. 1 b) and can be seen as the cause of the increased computational strength of $\Xi$, compared to that of $\Theta$. Even $\Theta$ will have some computational strength, though, as any instance of $\Theta$ will compute, in conjunction with $\mu$, a realiser of transfinite recursion, see $[11,13]$, and will in particular compute functions that are not hyperarithmetical. However, any function uniformly computable in all instances of $\Theta$ will be hyperarithmetical, see [13] for a proof.

Remark 1. Our interpretation of the Lindelöf property of the Baire space may seem a bit odd, since we actually start with a countable cover $\left\{B_{\bar{f}(F(f))} \mid f \in\right.$ $\left.\mathbb{N}^{\mathbb{N}}\right\}$. The original assumption in Lindelöf [9] was essentially as follows:

For $x \in E \subseteq \mathbb{R}^{n}$, let $D_{x}$ be a disc with centre in $x$ and a positive radius.

Then, selecting a countable set of such discs is equivalent to select a countable set of centres, so our formulation captures the original formulation of the Lindelöf property correctly. 
The easy proof of the Lindelöf property will use $\Sigma_{1}^{1}[F]$-comprehension and countable choice, and for proving that $\mathbb{R}^{n}$ is hereditarily Lindelöf, there does not seem to be any good alternatives. Our choice-free proof for $\mathbb{N}^{\mathbb{N}}$ can be seen as a special proof for the set of irrational numbers. It is well known that $\mathbb{N}^{\mathbb{N}}$ and the set of irrationals between 0 and 1 are homeomorphic via continued fractions.

\subsection{Non-monotone Inductive Definitions}

Let $F: 2^{\mathbb{N}} \rightarrow 2^{\mathbb{N}}$, where $2^{\mathbb{N}}$ denotes the powerset of $\mathbb{N}$, identified with the set of characteristic functions. We may view $F$ as a functional of type 2 , ignoring the necessary coding. We may also consider $F$ as an inductive definition:

Definition 2. Let $F: 2^{\mathbb{N}} \rightarrow 2^{\mathbb{N}}$, and let $\alpha$ be an ordinal number. By recursion on $\alpha$ we define

$$
\Gamma_{\alpha}(F)=\bigcup_{\beta<\alpha} F\left(\Gamma_{\beta}(F)\right) .
$$

The sequence $\Gamma_{\alpha}(F)$ indexed by ordinals is an increasing sequence of subsets of $\mathbb{N}$, with $\Gamma_{0}(F)=\emptyset$. For cardinality reasons, there is a countable ordinal $\alpha_{0}$, depending on $F$, such that $\Gamma_{\alpha_{0}}(F)=\Gamma_{\alpha_{0}+1}(F)$, i.e. $F\left(\Gamma_{\alpha_{0}}(F)\right) \subseteq \Gamma_{\alpha_{0}}(F)$.

Definition 3. We let $\Gamma$ be the functional of type 3 defined by $\Gamma(F)=\Gamma_{\alpha_{0}}(F)$ where $\alpha_{0}$, depending on $F$, is as above.

We observe that if we let $G(X)=F(X) \backslash X$ for $X \in 2^{\mathbb{N}}$, then $\Gamma_{\alpha}(F)=\Gamma_{\alpha}(G)$ for all $\alpha$, and in particular, $\Gamma(F)=\Gamma(G)$.

Remark 2. The functional $F$ is monotone if $A \subseteq B \Rightarrow F(A) \subseteq F(B)$. Our inductive definitions are not necessarily monotone, so we call them non-monotone in general. Non-monotone inductive definitions were in particular studied in the late 60's and early 70's, see e.g. Sect. III of [4] with papers by Stål Aanderaa, Douglas Cenzer, Robin O. Gandy and Wayne Richter/Peter Aczel.

\section{The Main Theorem}

Theorem 1. The five functionals are related as follows:

a) For any instance of $\Xi$, there is an instance of $\Theta$ uniformly computable in $\Xi$ and $\mu$.

b) $\mathrm{S}$ is uniformly computable in $\mu$ and any instance of $\Xi$.

c) $\Gamma$ is uniformly computable in $\mathrm{S}$ and any instance of $\Theta$.

d) There is an instance of $\Xi$ computable in $\Gamma$.

Proof. a) is easy. Computing a finite covering from a countable covering of $C$ is computable even in the Turing sense. Given a covering of $C$ we extend this to a covering of $\mathbb{N}^{\mathbb{N}}$ such that each $f \notin C$ is covered by an open set disjoint from $C$, and then $\Xi$ provides a countable sub-covering of the original covering of $C$.

b) is proved in [14], and can also be considered as an easy exercise. The proof 
of c), which is the main technical achievement of this note, will be given in a separate section, see below, so let us prove d).

We need the Kleene-Brouwer ordering: If $s$ and $t$ are finite sequences of integers, then $s \prec_{\mathrm{KB}} t$ if $s$ is an extension of $t$ or if $s$ is below $t$ in the lexicographical ordering. If $F: \mathbb{N}^{\mathbb{N}} \rightarrow \mathbb{N}$ and $A$ is a set of finite sequences $s$, we let $G(A)=\left\{\bar{f}\left(F\left(f_{A}\right)\right)\right\}$, where $f_{A}$ is the least function that properly bounds $\bigcup_{s \in A} B_{s}$, provided that this set is bounded in the lexicographical ordering of $\mathbb{N}^{\mathbb{N}}$. If not, we let $G(A)=\emptyset$.

Each set $\Gamma_{\alpha}(G)$ will be a set of finite sequences well-ordered by the KleeneBrouwer ordering, and if $\alpha<\beta$, then $\Gamma_{\beta}(G)$ will be an end extension of $\Gamma_{\alpha}(G)$ with respect to this ordering. Moreover, the union $\bigcup_{s \in \Gamma_{\alpha}(G)} B_{s}$ will be an initial segment $O_{\alpha}$ of $\mathbb{N}^{\mathbb{N}}$ under the lexicographical ordering of $\mathbb{N}^{\mathbb{N}}$. Finally, the function $f_{\alpha}=f_{\Gamma_{\alpha}(G)}$ is definable from $\Gamma(G)$ uniformly in the finite sequence $s_{\alpha}$ added at stage $\alpha$. Thus we may arithmetically extract an enumeration of the set $\left\{f_{\alpha} \mid\right.$ $\left.s_{\alpha} \in \Gamma(G)\right\}$, and this will be the output of our $\Xi(F)$.

Remark 3. Borel's proof in [2] essentially starts with an open covering of [0,1], builds up a larger and larger half-open interval $\left[0, x_{\alpha}\right)$ by, at each stage adding an open set containing $x_{\alpha}$. Borel assumes that the original covering is countable, but this is not needed in order to show that the process must stop at some countable ordinal. Finally, through backtracking the process, Borel uses that there are no infinite descending sequences of ordinals in order to extract the finite covering. The point is that Borel's construction is deterministic and choice free, the finite sub-covering is fully determined by the map $x \mapsto O_{x}$ through his proof.

Remark 4. We see that modulo computability relative to $\mu$ there is a minimal instance of $\Xi$, and this one is equivalent to $\Gamma$. On the other hand, the analysis of computability in $\Theta$ in $[11,13]$ shows that there is no minimal instance of $\Theta$. However, if one takes the instance $\Theta_{0}$ that is naturally obtained using Borel's proof, then $\mathrm{S}$ will be computable in $\Theta_{0}$ and $\mu$ (see [11]), and consequently, $\Theta_{0}$ is also equivalent to $\Gamma$ modulo $\mu$. A closer inspection of the proofs will show that we only need a tiny fragment of Kleene-computability to establish these equivalences, we do not need the scheme for primitive recursion and the scheme of enumeration.

\subsection{The proof of Thm. 1 c)}

We will see how we may compute the inductive closure $\Gamma(F)$ from $F, \Theta$ and $S$. So, let $F$ be given. For the sake of notational simplicity, we assume that $F(A)$ is disjoint from $A$ for all $A$.

Associated with the sequence $\Gamma_{\alpha}(F)$, we have the relation $\Delta(F)$ on $\Gamma(F)$ defined by $\Delta(F)(a, b)$ if the least $\beta$ such that $a \in \Gamma_{\beta}(F)$ is less than or equal to the least $\gamma$ such that $b \in \Gamma_{\gamma}(F)$. The relation $\Delta(F)$ is inductively definable as well, with approximations $\Delta_{\alpha}(F)$ defined on $\Gamma_{\alpha}(F)$.

To be more precise, we may use the operator $\tilde{F}$ to define $\Delta(F)$ as follows:

If $R$ is a binary relation, we let $D_{R}$ be the domain of $R$, here defined as the set of $a$ such that for some $b$, either $(a, b) \in R$ or $(b, a) \in R$. Then $\tilde{F}(R)$ will, 
by definition, consist of all pairs $(a, b)$ where $a \in D_{R}$ and $b \in F\left(D_{R}\right)$ together with all pairs $(a, b)$ where both $a$ and $b$ are in $F\left(D_{R}\right)$. Instead of defining $\Gamma(F)$ directly with the help of $\Theta$, we extract $\Delta(F)=\Gamma(\tilde{F})$ from $F, S, \mu$ and $\Theta$.

We now need to establish some notation and general machinery: The advantage of $\Delta(F)$ is that it is a prewellordering. A preordering is a binary relation $R$ that is both transitive and reflexive, and such that for all $a, b \in D_{R}$ we have $R(a, b)$ or $R(b, a)$ (or both). The corresponding strict relation will be $R_{\mathrm{s}}(a, b) \leftrightarrow$ $R(a, b) \wedge \neg R(b, a)$, and $a$ is in the well founded part $W_{R}$ if $a \in D_{R}$ and there is no $R_{\mathrm{s}}$-descending sequence starting with $a$.

If $R$ is a preordering and $a \in D_{R}$, we let $D_{R}[a]=\left\{b \in D_{R} \mid R_{\mathrm{s}}(b, a)\right\}$ and we let $[a]_{R}=\{b \mid R(a, b) \wedge R(b, a)\}$. These are the initial segments and layers (or equivalence classes) of $D_{R}$.

We will now see how we can code all this with functions $f: \mathbb{N} \rightarrow\{0,1\}$ :

Definition 4. a) Let $f: \mathbb{N} \rightarrow\{0,1\}$. Let $R_{f}(a, b)$ if $f(\langle a, b\rangle)=1$. We write $D_{f}$ for $D_{R_{f}}$.

b) We say that $f$ is in PREO if $R_{f}$ is a preordering.

c) If $f \in$ PREO and $a \in D_{f}$, we let $D_{f}[a]=D_{R_{f}}[a]$ and $[a]_{f}=[a]_{R_{f}}$

d) If $f \in \mathrm{PREO}$, we write $W_{f}$ for $W_{R_{f}}, R_{f}[a]$ for the restriction of $R_{f}$ to $D_{f}[a]$ and $R_{f}[W]$ for the restriction of $R_{f}$ to $W_{f}$.

We now link this to the inductive operator $F$, which we still assume to be fixed:

Lemma 1. Let $f \in \mathrm{PREO}$. The following are equivalent:

1. $W_{f}=\Gamma(F)$ and $R_{f}[W]=\Delta(F)$

2. i) For all $a \in W_{f}$ we have that $[a]_{f}=F\left(D_{f}[a]\right)$

ii) $F\left(W_{f}\right)=\emptyset$.

The proof is trivial.

Lemma 2. Let $F$ be as above. There is a type 2 functional $G:\{0,1\}^{\mathbb{N}} \rightarrow \mathbb{N}$, uniformly computable in $S$ and $F$, such that for any instance of $\Theta, \Theta(G)$ will contain an element $f$ such that $f \in$ PREO and such that $W_{f}$ and $R_{f}[W]$ either satisfy i) and ii) in Lemma 1, or has a proper initial segment that does so.

Proof. We define $G$ by cases, and, at the same time, explain what is achieved in that case. For each case, we assume that the previous cases do not apply. Whenever we write "let $a, b$ etc. be such and such", we can make the definition precise by applying numerical search, i.e. using $\mu$, which is computable in S.

The aim is to define $G$ such that whenever $G(f)>0$, then $\bar{f}(G(f))$ has no extension $g$ that is in PREO and such that $(\Gamma(F), \Delta(F))$ is equal to $\left(D_{g}, R_{g}\right)$, while when $G(f)=0$, we can define $(\Gamma(F), \Delta(F))$ from $f$, using $\mu, S$ and $F$. Thus, in order to induce a finite set of neighbourhoods covering the one $g$ coding $(\Gamma(F), \Delta(F)), \Theta(G)$ must contain an $f$ with $G(f)=0$, and from which we can compute $(\Gamma(F), \Delta(F))$ using $\mu$ and $\mathrm{S}$.

The first part of the aim is met by, for each $f$ with $G(f)>0$, letting $G(f)>\langle a, b\rangle$ for some pair $(a, b)$ such that $f(\langle a, b\rangle)=0 \Leftrightarrow \Delta(F)(a, b)$. 
1. If $f$ is not in PREO, there will be a number $n$ such that $\bar{f}(n)$ cannot be extended to any $f^{\prime} \in$ PREO. We let $G(f)=n$ for the least such $n$.

2. If $(\Gamma(F), \Delta(F))$ is all of, or an initial segment of $\left(D_{f}, R_{f}\right)$, then $G(f)=0$. This can be checked using $F, S$ and $\mu$, since if this is the case, it will be the case also for $\left(W_{f}, R_{f}[W]\right)$, and from this relation, all initial segments are arithmetically defined. This case clearly satisfies our aim.

3. For some $a \in W_{f}$, we have that $[a]_{f} \neq F\left(D_{f}[a]\right)$. Select an $R_{f}$-minimal such $a$. Then $\left(D_{f}[a], R_{f}[a]\right)$ is a proper initial segment of $(\Gamma(F), \Delta(F))$, proper since we are not in Case 2. There will be two subcases:

i) $[a]_{f} \subset F\left(D_{f}[a]\right)$. Since the sets are not equal, let $b$ be in $F\left(D_{f}[a]\right)$, but not in $[a]_{f}$. If $f(\langle b, b\rangle)=0$, it suffices to let $G(f)=\langle b, b\rangle+1$ to achieve our aim. If $f(\langle b, b\rangle)=1$, we have that $b \in D_{f}$ while $b$ is neither $R_{f^{-}}$ equivalent to, nor $R_{f}$-less than $a$, so we must have $f(\langle a, b\rangle)=0$. Since $a$ and $b$ appear at the same stage in the $F$-recursion, it suffices to let $G(f)=\langle a, b\rangle+1$.

ii) Otherwise. We may then as well assume that $a \notin F\left(D_{f}[a]\right)$. Since we are not in Case 2, there is a $b \in F\left(D_{f}[a]\right)$. If $f(\langle b, b\rangle)=0$, we may let $G(f)=\langle b, b\rangle+1$. If $f(\langle b, b\rangle)=1, b \in D_{f}$, and we will use that $R_{f}$ is a preordering. We must have that $f(\langle a, b\rangle)=1$, since $b \notin D_{f}[a]$. But we will not have $\Delta(F)(a, b)$ since $b \in D_{f}[a] \cup F\left(D_{f}[a]\right)$, while $a$ is not. Thus it suffices, in this case, to let $G f)=\langle a, b\rangle+1$.

4. In this remaining case, we have that $W_{f}$ is one of the initial segments of $\Gamma(F)$, but not $\Gamma(F)$ itself. Let $a \in F\left(W_{f}\right)$. If $f(\langle a, a\rangle)=0$, we let $G(f)=$ $\langle a, a\rangle+$,1 . This suffices since $\Delta(F)(a, a)$. If $f(\langle a, a\rangle)=1$, we use that $R_{f}$ is a preordering, $a \in D_{f}$, but $a$ is not in the well founded part of of $R_{f}$. In particular, there will be some $b$ not in $W_{f}$ such that $f(\langle b, a\rangle)=1$ while $f(\langle a, b\rangle)=0$. If we let $G(f)=\max \{\langle b, a\rangle,\langle a, b\rangle\}+1$, we have ensured that we are in conflict with $\Delta(F)$.

We have defined $G(f)$ for all $f$, and the construction of $G$ is such that if $G(f)>$ 0 , then either $f \notin \mathrm{PREO}$ or for some $(a, b)$ we have that $\langle a, b\rangle<G(f)$ and $f(\langle a, b\rangle)=0 \Leftrightarrow \Delta(F)(a, b)$. Thus we cannot cover the Cantor space just by neighbourhoods given by $\bar{f}(G(f))$ for $f$ with $G(f)>0$. Consequently, $\Theta(G)$ must contain some $f$ with $\Theta(f)=0$. We can compute $\Gamma(F)$ uniformly in any such $f$, using $\mu$ and $S$.

Thm. $1 \mathrm{c}$ ) is an immediate consequence of this lemma.

\section{Speculations on Functionals of Type 3}

Prior research on the computational strength of functionals of type 3 has, to our knowledge, been concentrated on the normal functionals (with ${ }^{3} E$ computable in them), the Superjump and continuous functionals like the fan functional and the Gandy-Hyland functional, also denoted $\Gamma$. Our project has exposed that the class of non-normal, countably based functionals of type 3 in which $\mu$ or ${ }^{2} E$ is computable contains objects that reflect actual mathematical strength in some 
sense. For instance, consequences of the uncountable Heine-Borel theorem HBU will have realisers of type 3 of their own, and the computational strength of these realisers may reflect, at least in some sense, the strength of these consequences.

The functional $\Lambda$, not discussed in this note, is also of this nature. $\Lambda$ will not be computable in any type 2 functional, and actually neither in the superjump, but it is strictly weaker than $\Theta$ in the sense that any instance of $\Theta$ computes an instance of $\Lambda$ while the converse does not hold, even modulo $\mu$.

One obvious challenge will be to classify the 1 -section of $\Gamma$, i.e. the class of functions computable in $\Gamma$. A reasonable conjecture is that this 1-section is generated in a gap-free manner, and that it corresponds to a class of functions computable by an Infinite Time Turing Machine, ITTM, within some time bound. The ITTM's were introduced by Hamkins and Kidder, but first appeared in published form in [5]. For a recent survey, see [17]. A more ambitious, but vague, conjecture is that there is some total, countably based functional $\Psi$ of type 3 such that Kleene-computations relative to $\Psi$ somehow reflects computations using ITTM's. For this to make sense, there should at least be a gap-structure in the 1-section of $\Psi$ resembling that of ITTM's. We know that there is a gapstructure for computations relative to ${ }^{3} E$, (see [10]), and by a Löwenheim-Skolem argument there will be countably based functionals with a similar structure, but beyond this, little is known. More is known about the gap-structure for ITTM's, see e.g. the recent [3].

\section{Acknowledgements}

I am grateful to Sam Sanders for initiating the project of which this note can be seen as an outcome, and for commenting fruitfully on the content of the note. I will also thank John P. Hartley for sharing his thoughts around the early days of countably based functionals with me. 


\section{Bibliography}

[1] Benno van den Berg, Eyvind Briseid and Pavol Safarik, A functional interpretation for nonstandard arithmetic, Ann. Pure and Appl. Logic 163 (2012), no 12, 1962 1994.

[2] Emile Borel, Sur quelques points de la théorie des fonctions, Ann. Sci. École Norm. Sup. (3) 12 (1895), 9 - 55.

[3] Merlin Carl, Bruno Durand, Gregory Lafitte ad Sabrina Ouazzani, Admissibles in Gaps, in Kari, Manea and Petre (eds.) Unveiling Dynamics and Complecity - CiE 2017, Springer LNCS 10307 (2017), 175 - 186.

[4] Jens Erik Fenstad and Peter G. Himan, Generalized Recursion Theory, NorthHolland/American Elsevier (1974).

[5] Joel D. Hamkins and Andy Lewis, Infinite Time Turing Machines, J. Symb. Log. 65, no. 2, 567 - 604 .

[6] John P. Hartley, Recursion on Countably Based Functionals, Thesis, University of Leeds (1982).

[7] John P. Hartley, The countably based functionals, J. Symb. Log. 48, no. 2, 458 474.

[8] John P. Hartley, Effective discontinuity and a characterisation of the superjump, J. Symb. Log. 50 (1985), no. 2, 349 - 358.

[9] Ernst Lindelöf, Sur Quelques Points De La Théorie Des Ensembles, Comptes Rendus (1903), 697 - 700.

[10] Johan Moldestad, Computations in Higher Types, Springer Lecture Notes in Mathematics 574 (1977).

[11] Dag Normann and Sam Sanders, Nonstandard Analysis, Computability Theory, and their connections, Submitted, Available from arXiv: https://arxiv.org. /abs/1702.06556 (2017).

[12] Dag Normann and Sam Sanders, The strength of compactness in Computability Theory and Nonstandard Analysis, Submitted, Available from arXiv: https:// arxiv.org/abs/1801.08172 (2018).

[13] Dag Normann and Sam Sanders, Nonstandard Analysis, Computability Theory, and Metastability, in preparation.

[14] Dag Normann and Sam Sanders, On the mathematical and foundational significance of the uncountable, submitted, available from arXiv: http://arxiv.org/ abs/1711.08939 (2017).

[15] Sam Sanders, The Gandy-Hyland functional and a computational aspect of Nonstandard Analysis, Computability, vol. 7, no. 1, pp. 7-43, 2018.

[16] Sam Sanders, Metastability and higher-order computability, Proceedings of LFCS18, Lecture Notes in Computer Science 10703, Springer.

[17] Philip D. Welch, Transfinite Machine Models, in Rod Downey (ed.) Turing's Legacy, ASL Lecture Notes in Logic 42, Cambridge University Press (2014), 493 -529 . 


\section{Appendix - Notes to the reviewers}

This note was written because I was invited to give a talk in the special session on continuous computation at CiE2018. Since what is continuous depends on the choice of topology, one may consider my contribution to be within the scope of the section.

Instead of writing an abstract that covers the content of my talk, I decided to build this note around one technical result that I have obtained as part of a project that is in cooperation with Sam Sanders. The actual talk will report to a larger extent on results from this project, hopefully with some further results obtained between now and late July. I am informed that the organisers of the session accept my choice of topic for my talk.

Dag Normann 